\title{
Transcranial Doppler detection of anterior cerebral artery vasospasm
}

\author{
Laura Lennihan, George W Petty, Matthew E Fink, Robert A Solomon, J P Mohr
}

\begin{abstract}
The performance of transcranial Doppler in the detection of anterior cerebral artery vasospasm and vasospasm in patients after subarachnoid haemorrhage was analysed. Transcranial Doppler and cerebral angiography were performed within the same 24 hours on each of 41 patients with acute subarachnoid haemorrhage. Sensitivity and specificity of transcranial Doppler to classify middle cerebral arteries, anterior cerebral arteries, and patients with angiographic vasospasm were determined at mean velocities of 120 and $140 \mathrm{~cm} / \mathrm{s}$. Accuracy of transcranial Doppler was better at 140 than at $120 \mathrm{~cm} / \mathrm{s}$. For the middle cerebral artery, sensitivity was $86 \%$, specificity $98 \%$. For the anterior cerebral artery, sensitivity was $13 \%$, specificity $100 \%$. Among all patients, sensitivity was $45 \%$, specificity $96 \%$. Among patients with anterior communicating artery aneurysms, sensitivity was $14 \%$, specificity $90 \%$. Therefore, transcranial Doppler accurately differentiates between middle cerebral arteries with and without vasospasm on angiography, but has a very low sensitivity for detecting anterior cerebral artery vasospasm and vasospasm in patients with anterior communicating artery aneurysms. Since vasospasm may involve anterior cerebral arteries while sparing middle cerebral arteries, especially after rupture of an anterior communicating artery aneurysm, caution should be exercised in using negative transcranial Doppler results to make treatment decisions based on the assumed absence of vasospasm.
\end{abstract}

\section{$(\mathcal{F}$ Neurol Neurosurg Psychiatry 1993;56:906-909)}

Transcranial Doppler (TCD) has been successfully applied to the detection of vasospasm following subarachnoid haemorrhage. Mean velocity in the middle cerebral artery (MCA) measured by TCD correlates well with vessel lumen diameter on angiography ${ }^{1-4}$ and is significantly higher in patients who develop delayed cerebral ischaemia compared with those who do not. ${ }^{5}$ TCD has good sensitivity and specificity for diagnosing MCA vasospasm. ${ }^{3467}$

Substitution of TCD for angiography to diagnose vasospasm of specific arteries could prove invaluable for research to determine the causes of vasospasm and delayed cerebral ischaemia, assess treatment of these conditions, and delineate predictors of outcome. Application of TCD to the clinical setting may assist in making decisions regarding timing of aneurysm surgery and selection and adjustment of therapy to alleviate vasospasm.

The accuracy of TCD in the diagnosis of MCA vasospasm has been encouraging with regard to both research and clinical issues. However, since the development of the TCD, it has been recognised that for the anterior cerebral artery (ACA), the anatomical course, most of it at right angles to the Doppler beam, and vascular interconnections may interfere with both measurement and interpretation ACA velocities by this non-invasive test. ${ }^{18-10}$ This report analyses the performance of the TCD test compared with angiography in the detection of ACA vasospasm.

\section{Methods}

During a 12 month period, all of the authors' patients with subarachnoid haemorrhage whose onset was within 14 days of admission to the Neurological Institute of New York were studied by TCD. The study includes all 41 patients who had a TCD within 24 hours of cerebral angiography and no surgical procedure between the Doppler and angiographic studies. No patient was included in this report more than once.

TCD examinations were performed with a pulsed, range-gated $2 \mathrm{mHz}$ TCD (EME TC2-64B, Eden Medizinische Elektronik $\mathrm{GmbH}$, Uberlingen, Germany) using the standard transtemporal technique to insonate and identify arteries. ${ }^{11}$ The maximum mean velocity and pulsatility index for each basal artery studied were recorded. On angiography, vasospasm was diagnosed if focal or diffuse narrowing of the MCA $M_{1}$, ACA $A_{1}$, or ACA $A_{2}$ segments was not explained by atherosclerosis or a congenital anomaly. TCD and angiography were performed and the results interpreted independently by reviewers having no knowledge of the patients' clinical status.

The sensitivity and specificity of TCD compared with cerebral angiography to diagnose vasospasm of a specific artery were determined at mean velocities of $>140 \mathrm{~cm} / \mathrm{s}$ and $>120 \mathrm{~cm} / \mathrm{s}$ for the MCA and ACA. In addition, the sensitivity and specificity of TCD to classify patients as having vasospasm were determined at these two mean velocities. 
Patients who for technical reasons had no arterial flow detected by TCD are not included in the analysis of TCD sensitivity and specificity but are reported in the description of TCD test results. Comparison of means of arterial mean velocities and comparison of means of pulsatility indices were performed using Student's $t$ test with $a=0 \cdot 05$.

\section{Results}

The study included 41 subjects, 28 women and 13 men with a mean age of 52 years (range 22-85). Thirty four had bilateral and seven unilateral carotid angiograms. All had bilateral TCD studies. Four patients (10\%) had no ultrasonic window at either temple; one patient had a left but not a right temporal window. Aneurysm locations are shown in table 1 .

Seventy five MCAs in 41 patients were studied angiographically and by TCD. An ultrasonic window was present in 66 and MCA flow was detected by TCD in all 66 . On angiography, seven of the 66 arteries were in spasm. Based on a mean velocity of $>140 \mathrm{~cm} / \mathrm{s}$ to diagnose vasospasm, TCD correctly classified six of the seven in spasm and 58 of the 59 not in spasm (sensitivity 86\%; specificity $98 \%$ ). Using a mean velocity of $>120 \mathrm{~cm} / \mathrm{s}$ to diagnose vasospasm, TCD sensitivity was unchanged and specificity dropped to $86 \%$ as a result of seven additional false positives. MCAs in spasm on angiogram had significantly higher mean velocities (mean $153 \mathrm{~cm} / \mathrm{s}$ ) than MCAs not in spasm (mean $72 \mathrm{~cm} / \mathrm{s}, \mathrm{p}<0.01$ ). Pulsatility indices were significantly lower in the MCAs in spasm (spasm mean 0.57 versus no spasm mean $1.04, \mathrm{p}<0.01)$.

Seventy five ACAs in 41 patients had both angiographic and TCD studies. One of these ACAs was aplastic. Nine arteries were not detected by TCD because there was no ultra-

\section{Table 1 Aneurysm locations}

\begin{tabular}{lr}
\hline Anterior communicating artery & 19 \\
Pericallosal artery & 3 \\
Posterior communicating artery & 10 \\
Middle cerebral artery & 1 \\
Basilar artery apex & 2 \\
Posterior cerebral artery & 1 \\
Posterior inferior cerebellar artery & 2 \\
No aneurysm & 3 \\
\hline
\end{tabular}

sonic window. TCD detected no flow in nine additional ACAs despite detection of flow in the ipsilateral MCA. On angiography, 15 of 66 ACAs studied by TCD were in spasm. Based on the $>140 \mathrm{~cm} / \mathrm{s}$ mean velocity criterion for vasospasm, TCD correctly classified only two of the 15 in spasm (sensitivity 13\%), 10 had mean velocities $<140 \mathrm{~cm} / \mathrm{s}$, and three had no flow detected. Using the $>140 \mathrm{~cm} / \mathrm{s}$ vasospasm criterion, TCD correctly classified all 51 ACAs not in spasm on angiogram (specificity 100\%). Changing the TCD vasospasm criterion to $>120 \mathrm{~cm} / \mathrm{s}$ did not improve the sensitivity, while two false positives lowered the specificity to $96 \%$. There was no significant difference between the mean velocities of those ACAs in spasm (mean $88 \mathrm{~cm} / \mathrm{s}$ ) versus those not in spasm (mean $66 \mathrm{~cm} / \mathrm{s}, 0.05<\mathrm{p}<0.10$ ) or between pulsatility indices of ACAs in spasm (mean $0 \cdot 83$ ) or not (mean $1 \cdot 01, \mathrm{p}>0 \cdot 10$ ).

Ten patients had vasospasm of one (four patients) or both (six patients) ACAs on angiogram (table 2). Seven had anterior communicating artery (ACom) aneurysms, three posterior communicating artery aneurysms. TCD correctly diagnosed vasospasm of the ACAs of only one patient. This patient had a posterior communicating artery aneurysm and diffuse, severe vasospasm affecting both middle and anterior cerebral arteries. Three other patients had vasospasm of both anterior and middle cerebral arteries, but TCD detected only the MCA vasospasm. Two of these patients had posterior communicating artery aneurysms, one an ACom aneurysm.

Six patients had vasospasm on angiography of ACAs, but not MCAs. All had ACom aneurysms. Ten of their 12 ACAs were in spasm, but five had spasm of only the postcommunicating ACA segment $\left(A_{2}\right)$, not the precommunicating ACA segment $\left(A_{1}\right)$. TCD failed to detect the vasospasm in all six patients and all nine ACAs in spasm that had ultrasonic windows.

Of the 37 patients with ultrasonic windows, 11 had vasospasm on angiography of at least one middle or anterior cerebral artery. At mean velocity $>140 \mathrm{~cm} / \mathrm{s}$, TCD sensitivity was $45 \%$ and specificity $96 \%$. All of the false negatives occurred in patients with ACom aneurysms. Among the 19 ACom aneurysm patients in the study, 17 had ultrasonic win-

Table 2 Comparison of angiographic and transcranial Doppler findings in patients with anterior cerebral artery vasospasm

\begin{tabular}{|c|c|c|c|c|c|c|c|c|c|c|}
\hline \multirow[b]{2}{*}{ Aneurysm } & \multicolumn{6}{|c|}{ Angiography vasospasm } & \multicolumn{4}{|c|}{ Transcranial Doppler velocity $(\mathrm{cm} / \mathrm{s})$} \\
\hline & $L M C A$ & $R M C A$ & $L A_{1}$ & $R A_{1}$ & $L A_{2}$ & $R A_{2}$ & $L M C A$ & $R M C A$ & $L A C A$ & $R A C A$ \\
\hline $\begin{array}{l}\text { ACOM } \\
\text { ACOM } \\
\text { ACOM } \\
\text { ACOM } \\
\text { ACOM } \\
\text { ACOM } \\
\text { ACOM } \\
\text { PCOM } \\
\text { PCOM } \\
\text { PCOM }\end{array}$ & $\begin{array}{l}\text { Yes } \\
\text { No } \\
\text { No } \\
\text { No } \\
\text { No } \\
\text { No } \\
\text { No } \\
\text { Yes } \\
\text { Yes }\end{array}$ & $\begin{array}{l}\text { Yes } \\
\text { No } \\
\text { No } \\
\text { No } \\
\text { No } \\
\text { No } \\
\text { No } \\
\text { Yes } \\
\text { Yes } \\
\text { No }\end{array}$ & $\begin{array}{l}\text { Yes } \\
\text { No } \\
\text { No } \\
\text { No } \\
\text { Yes } \\
\text { Yes } \\
\text { Yes } \\
\\
\text { Yes } \\
\text { Yes }\end{array}$ & $\begin{array}{l}\text { Yes } \\
\text { No } \\
\text { No } \\
\text { Yes } \\
\text { Yes } \\
\text { No } \\
\text { No } \\
\text { Yes } \\
\text { Yes } \\
\text { No }\end{array}$ & $\begin{array}{l}\text { Yes } \\
\text { Yes }\end{array}$ & $\begin{array}{l}\text { Yes } \\
\text { Yes } \\
\text { Yes }\end{array}$ & $\begin{array}{r}188 \\
122 \\
56 \\
106 \\
66 \\
34 \\
120\end{array}$ & $\begin{array}{r}86^{\star} \\
N^{*} \\
64 \\
72 \\
114 \\
50 \\
122 \\
162 \\
198 \\
166\end{array}$ & $\begin{array}{c}116^{\star} \\
42^{\star} \\
64^{\star} \\
114 \\
\mathrm{ND}^{\star} \\
20^{\star} \\
\mathrm{ND}^{\star}\end{array}$ & $\begin{array}{c}42^{\star} \\
N^{\star} \\
N^{\star} \\
96^{\star} \\
96^{\star} \\
42^{\star} \\
86 \\
78^{\star} \\
206 \\
110\end{array}$ \\
\hline
\end{tabular}

ACOM = anterior communicating artery; PCOM = posterior communicating artery; LMCA = left middle cerebral artery $R M C A=$ right middle cerebral artery; $L A_{1}=$ left $A_{1}$ segment, anterior cerebral artery; $R A_{1}=$ right $A_{1}$ segment, anterior cerebral artery; $\mathrm{LA}_{2}=$ left $\mathrm{A}_{2}$ segment, anterior cerebral artery; $\mathrm{RA}_{2}=$ right $\mathrm{A}_{2}$ segment, anterior cerebral artery; ND = no flow detected; $\mathrm{NW}=$ no ultrasonic window

$\star$ False negative transcranial Doppler. 
dows and seven of these 17 had vasospasm on angiography. At mean velocity $>140 \mathrm{~cm} / \mathrm{s}$, TCD had a sensitivity of $14 \%$ (one of seven) and specificity of $90 \%$ (nine of 10 ). The one ACom aneurysm patient with a positive TCD had MCA as well as ACA vasospasm; the six ACom patients with negative TCDs but positive angiograms had only ACA vasospasm.

\section{Discussion}

In this study, mean velocity measured by TCD had the high sensitivity and specificity for diagnosis of MCA vasospasm previously reported. ${ }^{3467}$ By contrast, TCD had a low sensitivity and high specificity for the detection of angiographically diagnosed ACA vasospasm. Also in this study, TCD had a low sensitivity for diagnosing vasospasm in patients with ACom aneurysms.

Most reports of TCD after subarachnoid haemorrhage have emphasised results of MCA insonation. Those studies which have also noted results of ACA insonation have found that ACA velocities add no information to MCA measurements, ${ }^{12}$ that there is a weak correlation between ACA diameter and ACA mean velocity, ${ }^{1-3}$ and that MCA vasospasm versus no vasospasm groups have significantly different maximum mean velocities but ACA vasospasm versus no vasospasm groups do not. ${ }^{7}$ Contributing to the low TCD sensitivity for detection of ACA vasospasm may be anatomy, test conditions and technique, and diagnostic criteria.

The course of the ACA $A_{1}$ segment makes transtemporal TCD detection of the $A_{1}$ more difficult than detection of the MCA. ${ }^{18-10}$ TCD may fail to provide a measure of velocity in more than $20 \%$ of $\mathrm{ACAs}^{110113}$ due to the frequency of no transtemporal ultrasonic window (4/41 patients in this study and $2 \cdot 7-10 \%$ of patients in other reports ${ }^{1491014}$ ) and the rate of failure to detect ACA flow even when there is a good MCA signal and no ACA vasospasm. ${ }^{1910} \mathrm{~A}_{1}$ hypoplasia or aplasia, affecting $5-12 \%$ of ACAs, ${ }^{15-17}$ may add to difficulty in detecting ACA flow.

The usual ACA $A_{1}$ course provides an angle of transtemporal TCD insonation which may result in an underestimate of mean velocity and therefore an underdiagnosis of $A_{1}$ vasospasm based on mean velocity criteria. Severe $A_{1}$ vasospasm which markedly reduces flow may increase the difficulty in locating and measuring $A_{1}$ flow. ${ }^{18}$ The ACA $\mathrm{A}_{2}$ segment is beyond the reach of transtemporal TCD so when vasospasm involves the $A_{2}$ but not the $A_{1}$ segment, TCD gives a false negative result. ACA vasospasm may be diagnosed in error when high ACA velocities reflect low vascular resistance distal to the point of insonation. For example, left internal carotid artery (ICA) occlusion may lead to increased flow, and therefore high velocities, in the right ACA if this artery becomes an important source of collateral blood flow to the left ICA territory.

TCD operator experience and skill are important for accuracy of vessel identification and velocity measurement. In this study, the accurate classification of MCA vasospasm by TCD makes it unlikely that poor TCD operator skill alone would be a sufficient explanation of the low TCD sensitivity for detecting ACA vasospasm.

The TCD diagnostic criteria for vasospasm tested in this study, $>120 \mathrm{~cm} / \mathrm{s}$ and $>140 \mathrm{~cm} / \mathrm{s}$ were selected based on reports of TCD performance at these velocity limits and the reported correlation between MCA velocity and lumen area. The MCA velocity criteria for vasospasm assessed by other investigators include $110 \mathrm{~cm} / \mathrm{s}$ (sensitivity $85 \%$, specificity $98 \%{ }^{3}$ ) and $120 \mathrm{~cm} / \mathrm{s}$ (sensitivity $59 \%$, specificity $100 \%,{ }^{4}$ and sensitivity $78 \%^{9}$ ) At $120 \mathrm{~cm} / \mathrm{s}$, TCD sensitivity to diagnose ACA vasospasm has been reported as $71 \% .^{9}$ In the MCA, mean velocity $>140 \mathrm{~cm} / \mathrm{s}$ correlates with reduction in lumen diameter to $<1.5 \mathrm{~mm}^{13}$, corresponding to $>75 \%$ reduction in lumen area. ${ }^{20}$ Because this degree of lumen stenosis significantly reduces blood flow, mean velocity $140 \mathrm{~cm} / \mathrm{s}$ has been recommended as the minimum velocity to diagnose vasospasm. ${ }^{3}$ Brain infarction ${ }^{5}$ and delayed ischaemic deficits ${ }^{2}$ due to vasospasm may be rare at velocities below $140 \mathrm{~cm} / \mathrm{s}$, whereas the incidence of ischaemia due to vasospasm rises at velocities above $140 \mathrm{~cm} / \mathrm{s.}^{27}$

In this study, mean velocity $>140 \mathrm{~cm} / \mathrm{s}$ proved a satisfactory TCD measure for diagnosing MCA vasospasm. Mean velocity $>140 \mathrm{~cm} / \mathrm{s}$ was a highly specific but very insensitive classifier of ACA vasospasm. Classification was no more accurate at mean velocity $>120 \mathrm{~cm} / \mathrm{s}$. Other measures, not examined in this study, such as comparison of ipsilateral internal carotid artery and ACA mean velocities and pulsatility indices, or determination of change in ACA velocities over time, may prove more accurate classifiers of ACA vasospasm.

Low TCD sensitivity to vasospasm in a patient despite high TCD sensitivity to MCA vasospasm has been reported previously and attributed to vasospasm affecting arteries other than the MCA stem. ${ }^{4}$ In this study, the patient characteristic which explained TCD insensitivity to vasospasm in a patient was aneurysm location, with all false negatives occurring in patients with ACom aneurysms. Anatomy and technical problems may contribute to this low sensitivity in patients with ACom aneurysms.

Vasospasm following rupture of ACom aneurysms is most likely to involve the ACAs. Although some patients with ruptured AComs will experience vasospasm of other arteries as well, others will have only ACA vasospasm $^{4}$ (and six of our seven patients with ACom aneurysms and vasospasm) and some will have vasospasm of the $A_{2}$ but not the $A_{1}$ segments $^{15}$ (and three of our seven patients with ACom aneurysms and vasospasm). As discussed above, TCD has a low sensitivity for both $A_{1}$ and $A_{2}$ vasospasm.

The condition of the patient affects the ease or difficulty of performing TCD. The 
restless, agitated patient may be unable to keep his head still long enough for accurate vessel identification and velocity measurement. The patient with a ruptured ACom aneurysm and consequent ACA vasospasm leading to frontal lobe ischaemia may be just the restless, agitated patient who cannot have a technically satisfactory TCD study.

In summary, the high specificities of TCD for classifying MCA and ACA vasospasm establish that the diagnosis of vasospasm can be made with confidence when mean velocities exceed $140 \mathrm{~cm} / \mathrm{s}$. Treatment decisions based on the presence of vasospasm, such as timing of surgery, institution of volume expansion, modification of calcium channel blocker dose, and selection of patients for percutaneous transluminal angioplasty of spastic cerebral arteries may be supported by such positive results and have been endorsed by other investigators. ${ }^{25792122}$ However, because TCD has such a low sensitivity for detecting ACA vasospasm and vasospasm in patients with ACom aneurysms, caution should be exercised in using negative TCD results (mean velocity $<140 \mathrm{~cm} / \mathrm{s}$ ) to make treatment decisions based on the assumed absence of vasospasm.

1 Aaslid R, Huber P, Nornes H. Evaluation of cerebrovascular spasm with transcranial Doppler ultrasound. $\mathcal{F}$ Neurosurg 1984;60:37-41.

2 Harders AG, Gilsbach JM. Time course of blood velocity changes related to vasospasm in the circle of Willis measured by transcranial Doppler ultrasound. $\mathcal{f}$ Neurosurg 1987;66:718-28.

3 Lindegaard KF, Nornes H, Bakke SJ, Sorteberg W, Nakstad P. Cerebral vasospasm after subarachnoid haemorrhage investigated by means of transcranial Doppler ultrasound. Acta Neurochir 1988;(Suppl)42 81-4.

4 Sloan MA, Haley EC, Kassell NF, et al. Sensitivity and specificity of transcranial Doppler ultrasonography in the diagnosis of vasospasm following subarachnoid hemorrhage; Neurology 1989;39:1514-8.

5 Seiler RW, Grolimund P, Aaslid R, Huber P, Nornes H. Cerebral vasospasm evaluated by transcranial ultraCerebral vasospasm evaluated by transcranial ultrasound correlated with clinical grade and CT-visualized
subarachnoid hemorrhage. $\mathcal{f}$ Neurosurg 1986;64:
594-600.

6 Compton JS, Redmond S, Symon L. Cerebral blood velocity in subarachnoid haemorrhage: a transcranial Doppler study. I Neurol Neurosurg Psychiatry 1987; 50:1499-503.

7 Sekhar LN, Wechsler LR, Yonas H, Luyckx K, Obrist W. Value of transcranial Doppler examination in the diagnosis of cerebral vasospasm after subarachnoid hemorrhage. Neurosurgery 1988;22:813-21.

8 Aaslid $R$, Huber P, Nornes H. A transcranial Doppler method in the evaluation of cerebrovascular spasm. Neuroradiology 1986;28:11-6.

9 Grolimund P, Seiler RW, Aaslid R, Huber P, Zurbruegg $H$. Evaluation of cerebrovascular disease by combined extracranial and transcranial Doppler sonography. Experience in 1039 patients. Stroke 1987;18:1018-24.

10 Hennerici M, Rautenberg W, Sitzer G, Schwartz A. Transcranial Doppler for the assessment of intracranial arterial flow velocity-part 1. Examination technique and normal values. Surg Neurol 1987;27:439-48.

11 Aaslid R, Markwalder TM, Nornes H. Noninvasive transcranial Doppler ultrasound recording of flow velocity in basal cerebral arteries. $\mathcal{F}$ Neurosurg 1982;57:769-74.

12 Hutchison $\mathrm{K}$, Weir B. Transcranial Doppler studies in aneurysm patients. Can $\mathcal{f}$ Neurol Sci 1989;16:411-6.

13 Arnolds BJ, Reutern GM. Transcranial Dopplersonography. Examination technique and normal reference values. Ultrasound Med Biol 1986;12:115-23.

14 Harders A, Gilsbach J. Transcranial Doppler sonography and its application in extracranial-intracranial bypass surgery. Neurol Res 1985;7:129-41.

15 Riggs HE, Rupp C. Variation in form of circle of Willis. The relation of the variations to collateral circulation: anatomic analysis. Arch Neurol 1963;8:8-14

16 Wollschlaeger G, Wollschlaeger PB, Lucas FV, Lopez VF. Experience and result with postmortem cerebral angiography performed as a routine procedure of the autopsy. Am $\mathcal{F}$ Roentgenol 1967;101:68-87.

17 Wollschlaeger PB, Wollschlaeger G. Anterior cerebral/ internal carotid artery and middle cerebral/internal carotid artery ratios. Acta Radiol Diag 1966;5:615-20.

18 Aaslid $R$, Huber $P$, Nornes $H$. Noninvasive transcranial Doppler ultrasound recording in basal cerebral arteries -a new approach to the evaluation of cerebrovascular spasm. In: Voth D, Glees P, eds. Cerebral vascular spasm. In: Voth D, Glees P, eds. Cerebral
spasm. Berlin: Walter de Gruyter, 1985:287-93.

19 Romner B, Ljunggren B, Brandt L, Saveland $H$. Correlation of transcranial Doppler sonography findings with timing of aneurysm surgery. F Neurosurg 1990; 73:72-6.

20 Gabrielsen TO, Greitz T. Normal size of the internal carotid, middle cerebral and anterior cerebral arteries. Acta Radiol 1970;10:1-10.

21 Konishi Y, Maemura E, Sato E, Hara M, Takeuchi K. A therapy against vasospasm after subarachnoidal haemorrhage: clinical experience of balloon angioplasty. Neurol Res 1990;12:103-5.

22 Seiler RW, Reulen HJ, Huber P, Grolimund P, Ebeling $U$, Steiger HJ. Outcome of aneurysmal subarachnoid hemorrhage in a hospital population: a prospective study including early operation, intravenous nimodipine, and transcranial Doppler ultrasound. Neurosurgery 1988;23:598-604. 